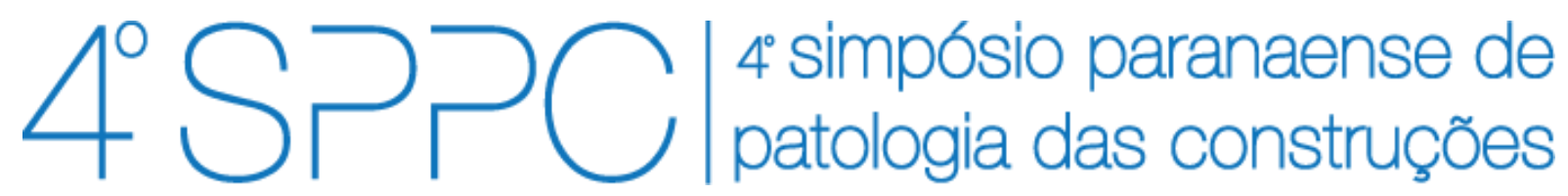

ISSN 2526-7248 artigo n. 4SPPC132, pp. 300-311, 2019

\title{
Previsão de vida útil de estruturas por modelos matemáticos e carbonatação natural
}

\author{
Nardino, Cladilson ${ }^{1}$; Valdés, Analiet Calvo²; Marcelo Henrique Farias de Medeiros ${ }^{3}$; \\ Ricardo Pieralisi ${ }^{4}$ \\ 1Doutorando, Universidade Federal do Paraná, clanardino@gmail.com \\ 2Doutoranda, Universidade Federal do Paraná, analietcv@yahoo.es \\ 3Doutor, Universidade Federal do Paraná, medeiros.ufpr@gmail.com \\ ${ }^{4}$ Doutor, Universidade Federal do Paraná, ricpieralisi@ufpr.br
}

\begin{abstract}
Resumo: A carbonatação do concreto é um processo físico-químico que envolve a difusão e reação de $\mathrm{CO}_{2}$ com produtos da hidratação do cimento. Essa reação reduz o $\mathrm{pH}$ do concreto o que impacta a durabilidade das estruturas. Com o auxílio de modelos matemáticos que consideram vários parâmetros que influenciam este mecanismo de deterioração, pode-se estimar a vida útil remanescente. $\mathrm{O}$ objetivo deste trabalho é correlacionar o avanço da frente de carbonatação, de forma natural, com a profundidade de carbonatação estimada a partir de modelos de vida útil, em corpos de prova armazenados numa sala com temperatura e umidades variáveis durante 7 anos. Os corpos de prova com menor relação água cimento $(\mathrm{a} / \mathrm{c})$ apresentaram menor profundidade de carbonatação. A amostra de referência, sem adição pozolânica e portanto com maior reserva alcalina, apresentou menores profundidade de carbonatação e não foi possível escolher um só modelo para representar o fenômeno.
\end{abstract}

Palavras chaves: carbonatação natural, frente de carbonatação, modelos de previsão.

\begin{abstract}
The carbonation of the concrete is a physical-chemical process that involves the diffusion and reaction of $\mathrm{CO}_{2}$ with products of the cement hydration. This reaction reduces the $\mathrm{pH}$ of the concrete which impacts the durability of the structures. With the aid of mathematical models that consider several parameters that influence this mechanism of deterioration, one can estimate the remaining useful life. The objective of this work is to correlate the advance of the carbonation front, naturally, with the carbonation depth estimated from life models, in test specimens stored in a room with temperature and variable humidity for 7 years. The specimens with lower water cement $(\mathrm{a} / \mathrm{c})$ showed lower depth of carbonation. The reference sample, without pozzolanic addition and therefore with higher alkaline reserves, presented lower carbonation depth and it was not possible to choose a single model to represent the phenomenon.
\end{abstract}

Keywords: natural carbonation, carbonation depth, life expectancy models. 
NARDINO, C; VALDÉS, A. C.; MEDEIROS, M.H.F.; PIERALISI, R. PREVISÃO DE VIDA ÚTIL DE ESTRUTURAS POR MODELOS MATEMÁTICOS E CARBONATAÇÃO NATURAL. $4^{\circ}$ Simpósio Paranaense de Patologia das Construções (40 SPPC), artigo 4SPPC132, pp. 300 - 311, 2019. DOI: 10.4322/2526-7248.052

\section{Introdução}

A carbonatação do concreto é um processo físico-químico que envolve a difusão de $\mathrm{CO}_{2}$ através dos poros de concreto e sua reação com produtos de hidratação como hidróxido de cálcio $\left(\mathrm{Ca}(\mathrm{OH})_{2}\right)$ e hidratos de silicato de cálcio [1, 2]. Essa reação reduz o $\mathrm{pH}$ da água dos poros do concreto de aproximadamente 12,5 para cerca de 9,0. Quando todo o $\mathrm{Ca}(\mathrm{OH})_{2}$ carbonata, o $\mathrm{pH}$ é reduzido para até 8,0 [3].

Logo, para que a reação descrita ocorra, os poros do concreto não podem estar secos nem saturados. Sendo a quantidade de água no concreto dependente da umidade do ar (U.R.), Helene (1986) [4] e Andrade (1992) [5] afirmam que as condições ideais de umidade relativa do ar para que ocorra a carbonatação devem estar entre 50 e $70 \%$ [3].

O tempo que a frente de carbonatação leva para alcançar o reforço de aço é conhecido como período de iniciação [6], e é uma fase importante da vida útil das estruturas de concreto armado. Vários métodos para prever este período foram propostos, incluindo modelos analíticos, modelos numéricos, e até mesmo redes neurais. Modelar a carbonatação é uma questão delicada, pois, embora vários fatores devam ser levados em conta, os usuários esperam que a pesquisa forneça modelos simples e fáceis de usar [7].

Um dos modelos mais usados para prever a profundidade de carbonatação é o modelo de Tuutti baseado na primeira lei de Fick. O modelo considera que a taxa de carbonatação é proporcional à raiz quadrada do tempo de exposição ao $\mathrm{CO}_{2}$. Entretanto, o coeficiente de carbonatação $(\mathrm{K})$, resume vários parâmetros, assumidos como constantes: concentração de $\mathrm{CO}_{2}$, quantidade de $\mathrm{CO}_{2}$ necessária para carbonatar um volume unitário de concreto e coeficiente de difusão para $\mathrm{CO}_{2}$ através de concreto carbonatado [6].

No entanto, em condições reais de exposição, a concentração de $\mathrm{CO}_{2}$ não é constante ao longo do tempo [8] e o coeficiente de difusão é sensível à umidade relativa e ciclos de umidade. Além disso, a quantidade de $\mathrm{CO}_{2}$ necessária para carbonatar um volume unitário de concreto depende da quantidade de $\mathrm{CO}_{2}$ disponível dentro dele [9]. Para concentrações regulares de $\mathrm{CO}_{2}$, somente os compostos de cimento hidratado reagem com o gás carbônico. Por outro lado, os compostos de cimento anidro reagem com o gás carbônico em altas concentrações de $\mathrm{CO}_{2}[10]$.

Diversos fatores afetam o processo de carbonatação, dessa forma a sintetização de todos esses fatores em um modelo simplificado se torna complexo [10]. Os fatores podem ser divididos em dois grandes grupos: fatores ambientais; e fatores intrínsecos ao concreto. Os fatores ambientais incluem: ocorrência de ciclos secos-úmidos [11]; umidade relativa; e concentração de $\mathrm{CO}_{2}$ [12]. Fatores intrínsecos estão relacionados principalmente à estrutura porosa do concreto, que influencia suas propriedades de transporte de massa, e à quantidade da substância que pode ser transformada em carbonatos [7].

Nesse contexto, o presente estudo tem como objetivo correlacionar o avanço da frente de carbonatação, de forma natural, com a aplicação de modelos de vida útil para estimar o tempo de vida útil residual da estrutura. Com o intuito de gerar dados que ajudem na extrapolação de forma confiável dos resultados obtidos nos testes laboratoriais para a modelagem de estruturas com base na durabilidade. 


\section{Modelos de previsão de vida útil}

Conforme exposto em Réus et al. [13], o modelo de Tutti [6], representado na Equação 1, é um dos primeiros modelos matemáticos desenvolvidos para prever o avanço da frente de carbonatação.

$$
X_{\mathrm{CO}_{2}}=k_{\mathrm{CO}_{2}} \times \sqrt{t}
$$

Onde: $\mathrm{X}_{\mathrm{CO} 2}$ é a espessura da profundidade carbonatada $(\mathrm{mm}) ; \mathrm{k}_{\mathrm{cO} 2}$ é o coeficiente de carbonatação $(\mathrm{mm} / \mathrm{Jano})$ e t é a idade em anos.

O modelo matemático desenvolvido por Possan [14], representado pela Equação 2, tem o objetivo de estimar a profundidade de carbonatação e a previsão da vida útil de projeto de estruturas de concreto (período de iniciação da corrosão). O modelo envolve variáveis de entrada de fácil obtenção como a resistência do concreto, o tipo de cimento, a umidade relativa média do ambiente onde a estrutura se encontra, entre outros.

$$
X_{C O_{2}}=k_{c}\left(\frac{20}{f_{c}}\right)^{k_{f c}} \cdot\left(\frac{t}{20}\right)^{\frac{1}{2}} \cdot \exp \exp \left[\left(\frac{k_{a d} \cdot a d^{\frac{3}{2}}}{40+f_{c}}\right)+\left(\frac{k_{c 02} \cdot C O_{2}^{\frac{1}{2}}}{60+f_{c}}\right)-\left(\frac{k_{U R} \cdot(U R-0,58)^{2}}{100+f_{c}}\right)\right] \cdot k_{c e}
$$

Onde: $X_{\mathrm{CO}_{2}}=$ profundidade de carbonatação média do concreto, em mm; $f_{c}=$ resistência característica à compressão axial do concreto, em MPa; $k_{c}=$ fator variável referente ao tipo de cimento empregado (Tabela 1 ); $k_{f c}=$ fator variável referente a resistência à compressão axial do concreto, em função do tipo de cimento utilizado; $t$ = idade do concreto, em anos; ad = teor de adição pozolânica no concreto, em \% em relação à massa de cimento; $k_{a d}=$ fator variável referente às adições pozolânicas do concreto - sílica ativa, metacaulim e cinza de casca de arroz - em função do tipo de cimento utilizado (Tabela 1); UR = umidade relativa média, em \% x 0,01; $k_{\cup R}=$ fator variável referente à umidade relativa, em função do tipo de cimento utilizado (Tabela 1); $\mathrm{CO}_{2}=$ teor de $\mathrm{CO}_{2}$ da atmosfera, em \%; $k_{\mathrm{CO} 2}=$ fator variável referente ao teor de $\mathrm{CO}_{2}$ do ambiente, em função do tipo de cimento utilizado; $k_{c e}=$ fator variável referente à exposição à chuva, em função das condições de exposição da estrutura.

Para a obtenção dos coeficientes do modelo em função das características do concreto e das condições ambientais, assim como dos coeficientes do modelo em função das condições de exposição, faz-se necessário o uso de tabelas fornecidas por Possan [14].

Outro modelo de vida útil empregado neste trabalho é o modelo $\mathrm{JCI}$ [15], que estima a espessura da camada carbonatada analisando apenas o tempo de exposição da estrutura e a relação a/c utilizada, conforme a Equação 3.

$$
X_{\mathrm{CO}_{2}}=\left(-0.357+0,9 \times \frac{a}{c}\right) \times \sqrt{t}
$$

Onde: $X_{\mathrm{CO}_{2}}=$ Espessura da profundidade carbonatada $(\mathrm{cm}) ; a / c=$ relação água/cimento e $\mathrm{t}=$ idade, em anos. 
Nota-se que este é um modelo muito simplificado em relação ao de Possan [14], ignorando efeitos importantes como a presença de adições, o teor de $\mathrm{CO}_{2}$ do ambiente e as condições de umidade.

Finalmente, através do modelo de carbonatação descrito na normativa espanhola Instrucción de Hormigón Estrutural (EHE-08) [16], pode-se determinar o período de tempo necessário para que se produza a carbonatação a uma profundidade em relação a superfície do concreto. Para isso, deve-se aplicar as Equações 4, 5 e 6.

$t_{i}=\left(\frac{X_{\mathrm{CO}_{2}}}{K_{c}}\right)^{2}$

Onde: $\mathrm{t}$ = tempo, em (anos); $X_{\mathrm{CO}_{2}}=$ profundidade, em $(\mathrm{mm})$ e $K_{c}$ coeficiente que leva em conta o ambiente, teor de ar incorporado e a resistência à compressão. $O K_{c}$ se determina através da Equação 5.

$K_{c}=C_{e n v} \times C_{\text {air }} \times a \times f_{c m}^{b}$

Sendo, $C_{e n v}$ coeficiente do ambiente; $C_{\text {air }}$ coeficiente de ar incorporado; a e b parâmetros em função do tipo de ligante e $f_{c m}$ a resistência média do concreto à compressão em MPa que pode ser estimada a partir da resistência característica especificada $\left(f_{c k}\right)$ através da Equação 6.

$f_{c m}=f_{c k}+8$

As tabelas com os coeficientes $C_{e n v}, C_{\text {air }}$ e os parâmetros a e b em função do tipo de ligante podem ser consultadas em (EHE-08) [16].

\section{Metodologia}

O programa experimental envolveu 6 traços de concreto que foram testados quanto ao parâmetro principal que foi a carbonatação natural. As variáveis de estudo são:

- Proporção de agregados: 2 níveis (1:5 e 1:6);

- Tipo de adição: 3 níveis (referência; Metacaulim; Metacaulim + Cal).

Desse modo, o experimento envolveu 6 casos de estudo. As variáveis de resposta do experimento são:

- Resistência à compressão aos 28 dias;

- Profundidade de carbonatação natural.

Os parâmetros fixados estão colocados a seguir:

- Cura: realizada por 28 dias em câmara úmida com $23 \pm 2$ oC e $100 \%$ de umidade relativa.

- Carbonatação natural: após o período de cura, as amostras foram armazenadas em sala com temperatura e umidade variáveis e teor de $\mathrm{CO} 2$ de aproximadamente $0,053 \%$, para a ocorrência da carbonatação natural. 


\subsection{Materiais}

\subsubsection{Aglomerantes}

Foi empregado o cimento CPV ARI com adição de 5\% de fíler calcário, tempo de pega maior ou igual a 1 hora, teor de óxido de magnésio menor que 6,5\% e resíduo insolúvel menor que $1 \%$ conforme especificações fornecidas pelo produtor. A análise química e física desse cimento é apresentada na Tabela 1.

Tabela 1: Características físico-químicas do cimento Portland CP V - ARI

\begin{tabular}{c|c|c|c|c|c|c|c|c|c}
\hline \multicolumn{10}{c}{ Análise química } \\
\hline $\mathrm{CaO}$ & $\mathrm{SiO}_{2}$ & $\mathrm{Al}_{2} \mathrm{O}_{3}$ & $\mathrm{Fe}_{2} \mathrm{O}_{3}$ & $\mathrm{MgO}$ & $\mathrm{SO}_{3}$ & $\begin{array}{c}\mathrm{CaO} \\
\text { livre }\end{array}$ & $\begin{array}{c}\text { Perda } \\
\text { Fogo }\end{array}$ & $\begin{array}{c}\text { Resíduo } \\
\text { Insolúvel }\end{array}$ & $\begin{array}{c}\text { Equivalente } \\
\text { Alcalino }\end{array}$ \\
\hline$(\%)$ & $(\%)$ & $(\%)$ & $(\%)$ & $(\%)$ & $(\%)$ & $(\%)$ & $(\%)$ & $(\%)$ & $(\%)$ \\
\hline 59,72 & 18,34 & 4,12 & 2,52 & 5,35 & 3,05 & 1,49 & 3,20 & 0,63 & 0,62 \\
\hline
\end{tabular}

\section{Análise Física}

\begin{tabular}{c|c|c|c|c|c|c|c|c}
\hline $\begin{array}{c}\text { Expansão } \\
\text { Quente }\end{array}$ & $\begin{array}{c}\text { Início } \\
\text { Pega }\end{array}$ & $\begin{array}{c}\text { Fim } \\
\text { Pega }\end{array}$ & $\begin{array}{c}\text { Consistência } \\
\text { Normal }\end{array}$ & Blaine & $\# 200$ & $\# 325$ & 7 dias & 28 dias \\
\hline$(\mathrm{mm})$ & $(\mathrm{h})$ & $(\mathrm{h})$ & $(\%)$ & $(\mathrm{cm} 2 / \mathrm{g})$ & $(\%)$ & $(\%)$ & $(\mathrm{MPa})$ & $(\mathrm{MPa})$ \\
\hline 0,50 & $02: 15$ & $03: 00$ & 27,6 & 4.370 & 0,10 & 2,80 & 42,70 & 51,10 \\
\hline
\end{tabular}

Foi utilizado metacaulim com massa específica igual a 2,56 kg/ $\mathrm{dm}^{3}, 0,1 \%$ de óxido de magnésio, e atividade pozolânica com o cimento (aos 7 dias) de 11,5\%, segundo a ficha técnica. A cal empregada foi classificada como cal hidratada dolomítica e sua análise química encontra-se na Tabela 2.

Tabela 2: Caracterização química da cal hidratada.

\begin{tabular}{l|l}
\hline Propriedade química & Quantidade \\
\hline Perda ao fogo $\left(95^{\circ} \mathrm{C}\right)(\%)$ & 17,1 \\
\hline Resíduo insolúvel (\%) & 2,2 \\
\hline Óxido de silício $\left(\mathrm{SiO}_{2}\right)(\%)$ & 9,3 \\
\hline Óxido de cálcio (CaO) (\%) & 44,9 \\
\hline Óxido de magnésio (MgO) (\%) & 30,3 \\
\hline Óxidos totais na base de não voláteis (CaO - MgO) $(\%)$ & 90,7 \\
\hline Anidrido carbônico (na fábrica) (\%) & 1,8 \\
\hline
\end{tabular}




\subsubsection{Agregados}

Como agregado graúdo foi utilizada uma composição de brita 1 e brita 0 na proporção 1:1 com mineralogia granítica. $O$ agregado miúdo foi uma areia natural retirada de leito de rio, com granulometria entre 0,06 e 0,2 mm.

\subsubsection{Concretos}

A Tabela 3 mostra os dados dos concretos usados no experimento. Deve-se destacar que a relação água/cimento foi ajustada de modo a produzir concretos enquadrados na mesma faixa de consistência que foi de $8 \pm 1 \mathrm{~cm}$ medida com o abatimento do tronco de cone. O metacaulim foi usado como substituição parcial do cimento com teor de $8 \%$ e a cal foi usada como adição com teor de $5 \%$ em relação ao total de ligante (cimento Portland + Metacaulim).

Tabela 3: Proporcionamento dos concretos.

\begin{tabular}{|c|c|c|c|c|c|c|c|c|}
\hline \multirow[b]{2}{*}{ Identificação } & \multirow[b]{2}{*}{ Quant. } & \multicolumn{3}{|c|}{ Aglomerante } & \multicolumn{3}{|c|}{ Agregados } & \multirow{2}{*}{$\begin{array}{c}\text { Relação } \\
\text { a/c }\end{array}$} \\
\hline & & $\begin{array}{l}\text { Cimento } \\
\text { Portland }\end{array}$ & Cal & Metacaulim & Areia & $\begin{array}{c}\text { Brita } \\
1\end{array}$ & $\begin{array}{c}\text { Brita } \\
0\end{array}$ & \\
\hline REF 1:5 & 2 & 1,0 & 0,0 & 0,0 & 2,18 & 1,41 & 1,41 & 0,61 \\
\hline REF 1:6 & 1 & 1,0 & 0,0 & 0,0 & 2,71 & 1,65 & 1,65 & 0,66 \\
\hline MET 1:5 & 2 & 0,92 & 0,0 & 0,08 & 2,18 & 1,41 & 1,41 & 0,66 \\
\hline MET 1:6 & 2 & 0,92 & 0,0 & 0,08 & 2,71 & 1,65 & 1,65 & 0,75 \\
\hline CAL 1:5 & 2 & 0,92 & 0,05 & 0,08 & 2,18 & 1,41 & 1,41 & 0,65 \\
\hline CAL 1:6 & 2 & 0,92 & 0,05 & 0,08 & 2,71 & 1,65 & 1,65 & 0,74 \\
\hline
\end{tabular}

Aos 28 dias de cura, o ensaio de resistência à compressão foi realizado de acordo com a NBR 5739 [17]. Conforme dados dos trabalhos desenvolvidos anteriormente, os três tipos de concreto representam um concreto com resistência à compressão $\left(f_{c k}\right)$ de $30 \mathrm{MPa}$.

\subsection{Métodos}

\subsubsection{Medida de profundidade de carbonatação}

O avanço da frente de carbonatação foi mensurado utilizando um indicador químico à base de fenolftaleína $(1 \%)$ de acordo com o procedimento indicado no documento RILEM TC-CPC 18/88. Este método consiste em aspergir a solução alcoólica na superfície recém fraturada da amostra. A região alcalina apresenta a coloração vermelho carmim, enquanto a região de $\mathrm{pH}$ reduzido (carbonatada) não apresenta coloração. A Figura 1 mostra os corpos de prova recém-fraturados e com a solução indicadora aplicada. Em cada corpo de prova foi efetuada a medição em 10 pontos utilizando-se um paquímetro digital com resolução de $0,01 \mathrm{~mm}$. 


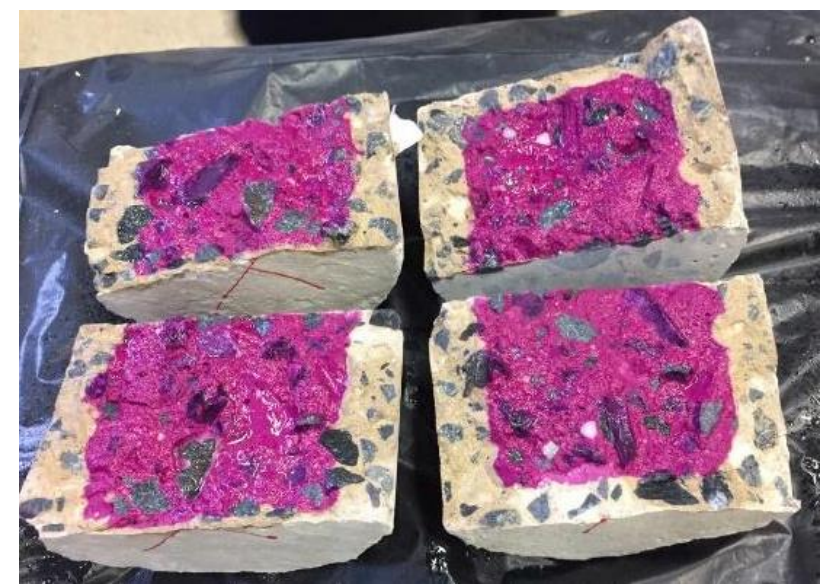

Figura 1: Teste colorimétrico

\subsubsection{Ensaio de carbonatação natural e previsão do avanço da frente de carbonatação}

O monitoramento da carbonatação natural foi baseado em medições de profundidade de carbonatação nas idades de 20, 32, 40, 77 e 94 meses. As medidas ocorreram em dois corpos de prova irmãos, moldados da mesma betonada e expostos em ambiente protegido da chuva com teor de $\mathrm{CO}_{2}$ de $0,053 \%$.

Utilizando os modelos de previsão de vida útil estimou-se a profundidade de carbonatação natural aos 94 meses. Os resultados estimados foram comparados com o valor de profundidade de carbonatação natural registrado no começo desse estudo, para definir qual dos modelos considerados no estudo se aproximava mais da medida real. Também se estimou o período de vida útil restante como consequência do fenômeno de carbonatação natural.

\section{Resultados e discussão}

A Figura 2, apresenta os resultados de profundidade de carbonatação natural para as amostras de referência, com metacaulim e com metacaulim e cal. A partir dos 40 meses de exposição, notou-se que nas amostras com o traço (1:6) obtiveram as maiores profundidades de carbonatação, quando comparadas com as amostras com o traço (1:5). Isso pode ter ocorrido porque os traços com menor consumo de cimento e maior relação $\mathrm{a} / \mathrm{c}$ apresentaram maiores valores de absorção de água por capilaridade. O que indica que esses concretos possuem uma rede de poros mais interconectada, facilitando o ingresso e difusão do $\mathrm{CO}_{2}$ para o interior dos poros do concreto.

Comparando os traços de mesma relação cimento:agregado e diferentes adições (referência, metacaulim e metacaulim mais cal), observou-se que o traço de referência apresentou menores profundidade de carbonatação em todos os casos. Isso ocorre em função da amostra de referência possuir maior reserva alcalina, visto que o metacaulim consome o hidróxido de cálcio da pasta de cimento, assim como comprovado no estudo de Medeiros et al. [18] e Hoppe Filho et al. [19]. 


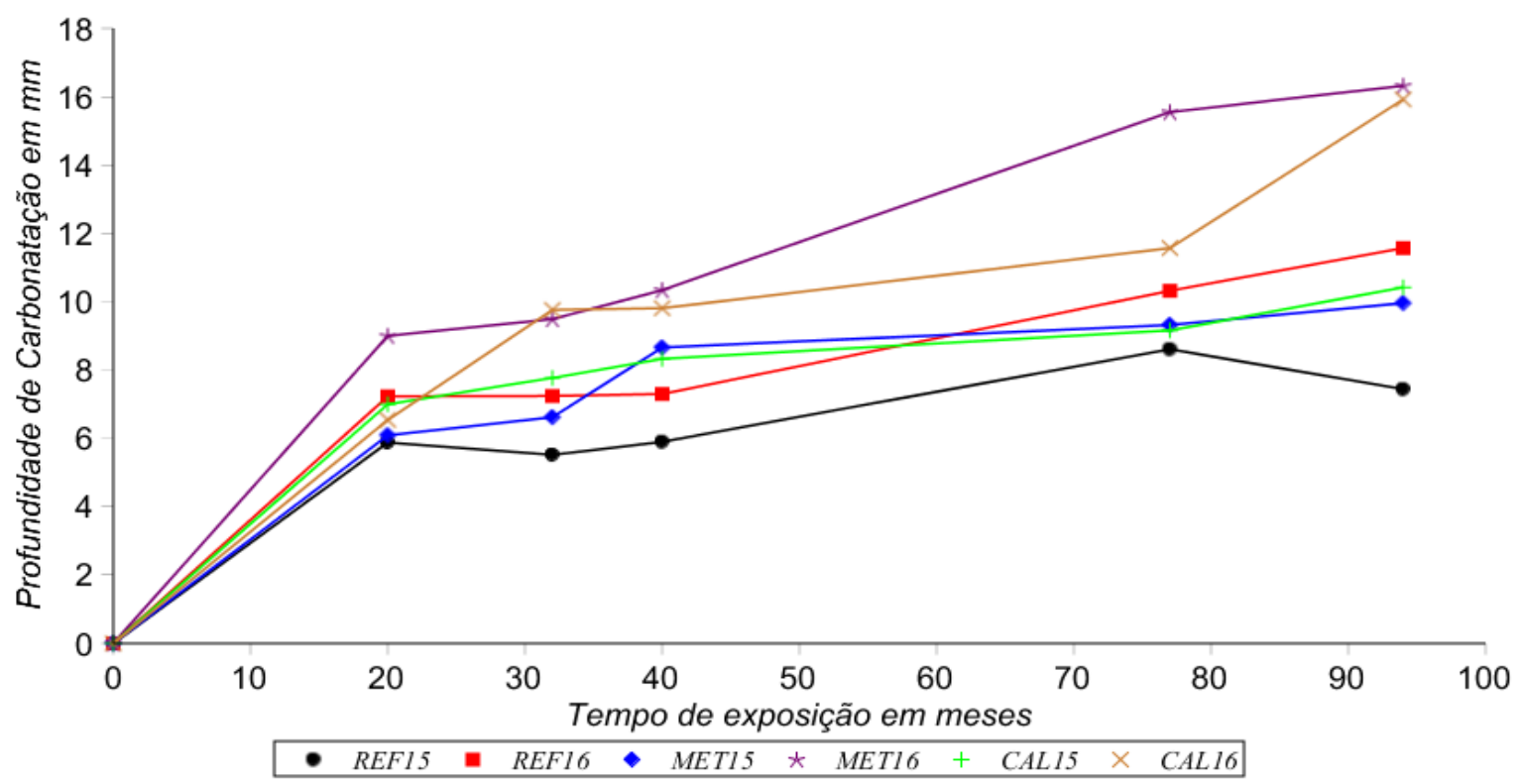

Figura 2: Profundidade de carbonatação natural das amostras.

Aos 40 meses, o concreto com 5\% de adição de cal (CAL 1:5 e CAL 1:6) apresentou valores muito próximos ao concreto com adição de 8\% metacaulim (MET 1:5 e MET 1:6). Aos 77 meses, conforme Reús et al. [20], nesses corpos de prova (5\% cal e 8\% metacaulim) se obtiveram resultados intermediários entre o traço de referência sem adição e o com 8\% de metacaulim. Aos 94 meses, as amostras de referência com o traço 1:5 (REF1:5) apresentaram uma redução na profundidade de carbonatação com relação à profundidade de carbonatação obtidas nessas amostras aos 77 meses. Os resultados observados até os 94 meses, indicam que a porcentagem de $5 \%$ de cal, não foi suficiente para compensar por completo a diminuição da reserva alcalina causada pelo metacaulim.

De acordo com os modelos de previsão de vida útil apresentados anteriormente (Equações 1, 2 e 3), estimou-se a profundidade carbonatação das amostras para um período de 94 meses (7,83 anos). Esses estão na Tabela 4.

Tabela 4: Estimativa da profundidade de carbonatação aos 94 meses (7,83 anos).

\begin{tabular}{c|c|c|c|c}
\hline \multirow{2}{*}{ Amostra } & \multicolumn{4}{|c}{ Espessura carbonatada (mm) } \\
\hline & Medido & Tutti, 1982 & Possan, 2010 & JCl, 2013 \\
\hline REF 1:5 & 7,44 & 3,96 & 3,96 & 11,79 \\
\hline REF 1:6 & 11,57 & 11,27 & 5,25 & 13,05 \\
\hline MET 1:5 & 9,96 & 6,37 & 3,92 & 13,05 \\
\hline MET 1:6 & 16,32 & 8,83 & 5,68 & 15,32 \\
\hline CAL 1:5 & 10,42 & 6,43 & 4,03 & 12,80 \\
\hline CAL 1:6 & 15,92 & 11,28 & 5,84 & 15,07 \\
\hline
\end{tabular}

Para conseguir aplicar o modelo de previsão Possan [14] foi assumido que a umidade relativa do local de armazenamento (UR) foi de 80,7\%, conforme Réus et al. [13]. 
Tendo em conta que o local de armazenamento é um ambiente interno protegido da chuva, o coeficiente $\mathrm{k}_{\mathrm{ce}}$ é 1,30 .

O modelo de $\mathrm{JCl}$ [15] mostrou-se o mais conservador, visto que esse modelo apresentou os maiores resultados de profundidade de carbonatação estimada. Por outro lado, o modelo de Possan [14] apresentou os menores resultados. Posteriormente, comparou-se esses valores de previsão da Tabela 4, com a medida efetivamente obtida aos 94 meses. A partir dessa análise foi possível constatar que 0 modelo de Tutti (1982) se aproximou $50 \%$ das vezes dos valores reais e o modelo $\mathrm{JCl}$ (2013) os outros 50 \%, aproximadamente. De acordo com Réus et al. [20], o modelo $\mathrm{JCl}$ [21] se aproximou $59 \%$ das vezes dos valores de carbonatação medidos aos 32, 44 e 77 meses. Porém, em alguns casos, os outros dois modelos (Eq. 1 e 3) se assemelham mais a realidade.

Com o intuito de representar o descrito anteriormente, foi determinado o coeficiente de correlação $\mathrm{R}^{2}$ apresentado na Tabela 5, com o objetivo de analisar a convergência dos dados obtidos, o que permite plotar curvas comparativas entre os modelos de previsão de vida útil e a medição real de cada amostra. Para isso, deve-se utilizar uma redução quadrática, para representar melhor o comportamento de uma curva característica.

Tabela 5: Valores de $\mathrm{R}^{2}$ em relação aos valores medidos.

\begin{tabular}{c|c|c|c|c|c|c}
\hline Amostra & REF 1:5 & REF 1:6 & MET 1:5 & MET 1:6 & CAL 1:5 & CAL 1:6 \\
\hline$R^{2}$ & 0,7391 & 0,9819 & 0,7924 & 0,987 & 0,9251 & 0,7863 \\
\hline
\end{tabular}

A partir dessa análise, foi possível constatar que os modelos de previsão de vida útil se aproximaram mais dos resultados obtidos nos corpos de prova com o menor consumo de cimento nos traços de referência e no $8 \%$ de metacaulim. Entretanto, no traço com $5 \%$ cal e $8 \%$ metacaulim, os maiores $\mathrm{R}^{2}$ se obtiveram no traço com o maior consumo de cimento (1:5).

Finalmente, de acordo com os modelos de previsão de vida útil apresentados anteriormente (Equações 1, 2, 3 e 4), estimou-se a vida útil remanescente das amostras estudadas como consequência do fenômeno de carbonatação natural durante 94 meses (7,83 anos). Para isto, se considerou que as amostras representam uma estrutura real projetada para atingir um tempo de vida útil de 50 anos, com 40 mm de cobrimento que corresponde à Classe II de acordo com a NBR 6118 - 2018.

Para aplicar o modelo EHE-08, os coeficientes $C_{\text {env }}$ e $C_{\text {air }}$ são iguais a 1, pois o local de armazenamento é um ambiente interno protegido da chuva e nenhum dos concretos tem ar incorporado. Como descrito anteriormente a resistência à compressão $\left(f_{c k}\right)$ dos concretos é de $30 \mathrm{MPa}$ e o cimento utilizado foi o CP-V-ARI que corresponde com o CEM I, portanto, o parâmetro "a" é igual a 1800 e o "b" a -1,7.

Na Tabela 6 é possível observar que através do modelo de previsão de vida útil Tutti (1982) se obtiveram os maiores tempos. Os concretos de referência apresentaram os maiores tempos de vida útil remanescente, se comparados com os concretos com adições com a mesma relação cimento:agregado. Nesse sentido, é importante 
destacar que o traço de referência apresentou as menores profundidades de carbonatação natural ao longo dos 94 meses (7,83 anos).

De fato, pode-se estabelecer uma correlação razoável entre os coeficientes de carbonatação e os parâmetros químicos (teor alcalino total, alcalinidade de reserva e $\mathrm{pH}$ ), conforme Shah e Bishnoi [22]. Nita e John [23], expõem que em uma pasta de cimento com pozolana, parte ou todo o hidróxido de cálcio é consumido por reações com a pozolana, o que normalmente provoca uma redução na intensidade dos picos correspondentes. Isto foi comprovado posteriormente por Medeiros et al. [18]. Nesse último trabalho, o aumento na velocidade de carbonatação provocado pelas adições pozolânicas foi de $32 \%$.

No entanto, não foi possível escolher só um dos modelos para representar o processo de carbonatação natural devido a variabilidade do processo. Vale destacar que na prática, é quase impossível que um modelo de previsão considere todas as condições reais já que diversos fatores afetam o processo de carbonatação natural. Além disso, o mercado espera que a pesquisa forneça modelos simples e fáceis de usar, o que diminui o número de fatores que um modelo pode considerar.

Tabela 6: Tempo de vida útil restante estimado a partir dos modelos de previsão de vida útil.

\begin{tabular}{c|c|c|c|c}
\hline \multicolumn{5}{c}{ Tempo restante (anos) } \\
\hline Amostra & Tutti (1982) & Possan (2010) & JCl (2013) & EHE -08 \\
\hline REF 1:5 & 38,82 & 44,76 & 43,91 & 45,98 \\
\hline REF 1:6 & 42,81 & 35,66 & 29,24 & 40,29 \\
\hline MET 1:5 & 40,46 & 34,05 & 41,21 & 42,8 \\
\hline MET 1:6 & 28,05 & 18,58 & 28,39 & 30,67 \\
\hline CAL 1:5 & 31,18 & 19,77 & 30,91 & 42,12 \\
\hline CAL 1:6 & 34,41 & 15,7 & 23,46 & 31,61 \\
\hline
\end{tabular}

\section{Conclusões}

As amostras de referência (sem adição de metacaulim) apresentaram menores profundidades de carbonatação. A adição de $5 \%$ de cal na dosagem do cimento reduziu o efeito de aumento da carbonatação causado pelo uso de $8 \%$ de metacaulim em substituição parcial do cimento Portland, porém, não foi suficiente para compensar completamente o efeito de aceleração da carbonatação resultado da adição de metacaulim. É necessário testar outros teores de cal, de forma a entender a sua ação nos concretos com adição de metacaulim.

Com os modelos utilizados neste trabalho, bem como as equações características de extrapolação do tempo de vida útil da estrutura, pode-se trabalhar com duas metodologias: previsão do cobrimento necessário para uma estimativa de vida útil determinada; ou a previsão de vida útil restante para a estrutura de acordo com as condições já existentes. Ambas as possibilidades permitem ações práticas e estimativas mais realistas de dimensionamento. 
Finalmente, não é possível assegurar que há só um modelo de previsão de vida útil que represente melhor a realidade, entre os modelos estudados. Sendo assim, se faz necessário escolher o modelos de previsão de vida útil que melhor se adapte às condições reais de exposição nas quais a estrutura estará inserida. Dessa forma, em conjunto com os ensaios de carbonatação, os modelos podem contribuir com a concepção de estruturas mais eficientes e preparadas para as degradações naturais que ocorrem. Ainda, permitem prever as frentes de carbonatação de estruturas reais já construídas a partir da caracterização do concreto utilizado, logo, possibilitando a realização de medidas preventivas ou de recuperação da estrutura.

\section{Referências}

[1] Castellote, M., Andrade, C., Turrillas, X., Campo, J., Cuello, G.J. (2008) "Accelerated carbonation of cement pastes in situ monitored by neutron diffraction". Cem Concr Re;38, pp.1365-73.

[2] Bertos, M.F., Simons, S.J.R., Hills, C.D., Carey, P.J. (2004), "A review of accelerated carbonation technology in the treatment of cement-based materials and sequestration of CO2". J Hazard Mater, B112, pp.193-205.

[3] Ferreira, A., Pereira, E., Raisdorfer, J.W., Medeiros, M.H.F. de, Klein, N.S., Capítulo 7: "Efeito da carbonatação na durabilidade do concreto armado",

[4] Helene, P.R.L.“Corrosão em armaduras para concreto armado”. São Paulo: PINI - IPT, 46 p. 1986.

[5] Andrade, C."Manual para diagnóstico de obras deterioradas por corrosão de armaduras". São Paulo: Pini. 1992

[6] Tuutti, K. "Corrosion of steel in concrete", Stockholm: Swedish Cement and Concrete Research Institute. 1982.

[7] Neves, R., Branco, F., de Brito, J., "Field assessment of the relationship between natural and accelerated concrete carbonation resistance". Cement \& Concrete Composites 41, pp. 9-15. 2013

[8] Santaguida, R., De Nile, F., Lauria, L., De Luca, G., Proietti, A., Carboni, G. "CO2 measurements at the sites of the Italian observing network". In: 13th WMO/IAEA, meeting of experts on carbon dioxide concentration and related tracers measurement techniques, Boulder, USA, September, pp. 156-60. 2005.

[9] Castellote, M., Fernandez, L., Andrade, C., Alonso, C. "Chemical changes and phase analysis of OPC pastes carbonated at different CO2 concentrations", Materials and Structures, Vol. 42, No. 4, pp. 515-525, 2009. doi:10.1617/s11527008-9399-1 RILEM.

[10] Sanjuán, M., Andrade, C., Cheyrezy, M. (2003) "Concrete carbonation test in natural and accelerated conditions". Revista in Advances in Cement Research, Vol. 15, No. 4, pp. 171-180, 2003. DOI: 10.1680/adcr.2003.15.4.171. 
NARDINO, C; VALDÉS, A. C.; MEDEIROS, M.H.F.; PIERALISI, R. PREVISÃO DE VIDA ÚTIL DE ESTRUTURAS POR MODELOS MATEMÁTICOS E CARBONATAÇÃO NATURAL. $4^{\circ}$ Simpósio Paranaense de Patologia das Construções (40 SPPC), artigo 4SPPC132, pp. 300 - 311, 2019. DOI: 10.4322/2526-7248.052

[11] Bakker, R. "Initiation period, In: Corrosion of steel in concrete". London: Chapman \& Hall, pp. 22-5. 1988.

[12] Verbeck, G. (1958), "Carbonation of hydrated Portland cement". In: Special Technical Publication 205. ASTM: USA, pp. 17-36. DOI: 10.1520/STP39460S.

[13] Possan, E. "Modelagem da carbonatação e previsão de vida útil de estruturas de concreto em ambiente urbano". 265 p. Tese (Tese) — Universidade Federal do Rio Grande do Sul, 2010.

[14] Han, et al., "Evaluation of concrete durability due to carbonation in harbor concrete structures". Journal Construction and Building Materials. 2013

[15] Instrucción del Hormigón estrutural (EHE - 08). Série normativa. Con comentarios de los miembros de la Comisión permanente de Hormigón. Gobierno de Espana. Ministério del Fomento. 2011

[16] ASSOCIAÇÃO BRASILEIRA DE NORMAS TÉCNICAS. "ABNT NBR 5739": Concreto - Ensaios de compressão de corpos-de-prova cilíndricos. Rio de Janeiro, 2007

[17] Medeiros, M. H. F.; Raisdorfer, J. W.; Hoppe Filho, J. "Influência da sílica ativa e do metacaulim na velocidade de carbonatação do concreto: relação com resistência, absorção, reserva alcalina e relação a/c". Ambiente Construído (Online), 2017.

[18] Hoppe Filho, J.; Gobbi, A.; Pereira, E.; Quarcioni, V. A.; Medeiros, M. H. F. Atividade pozolânica de adições minerais para cimento Portland (Parte I): Índice de atividade pozolânica (IAP) com cal, difração de raios-X (DRX), termogravimetria (TG/DTG) e Chapelle modificado. Materia-Rio de Janeiro, v. 22, p. 1-18, 2017.

[19] Réus, Giovana Costa; Ditzel, Luiz Felipe S.; Medeiros, Marcelo H. F. "Efeito do metacaulim na carbonatação natural: avaliação com modelos de previsão de vida útil". In: Conferência Nacional de Patologia e Recuperação de Estruturas (CONPAR). Recife, Pernambuco. 30 e 31 de agosto de 2017.

[20] Revert, Andres Belda; De Weerdt, Klaartje; Hornbostel, Karla; Geiker, Mette Rica. Carbonation-induced corrosion: Investigation of the corrosion onset. Revista Construction and Building Materials. Volume 162, 20 February 2018, Pages 847-856

[21] Shah Vineet; Bishnoi, Shashank. Carbonation resistance of cements containing supplementary cementitious materials and its relation to various parameters of concrete. Revista Construction and Building Materials. Volume 178, 30 July 2018, Pages 219-232

[22] Nita, C.; John, V. M., Materiais Pozolânicos: o metacaulim e a sílica ativa. Boletim Técnico da Escola Politécnica da USP, BT/PCC/451, pp. 1-13, 2007. 